\title{
Multiphasic disseminated encephalomyelitis presenting as alternating hemiplegia
}

\author{
Sonia Khan, Basim A Yaqub, Charles M Poser, Saleh M Al Deeb, Saeed Bohlega
}

\begin{abstract}
Two cases of alternating hemiparesis are reported, one in a black Sudanese woman, the other in a Saudi man, who had two episodes of alternating hemiparesis separated in time by six and three years respectively. Based on the typical appearance of the MRI and the results of brain biopsy, the diagnosis of multiphasic disseminated encephalomyelitis was made rather than that of multiple sclerosis. This entity is also differentiated from recurrent disseminated encephalomyelitis, where the relapses are symptomatically stereotyped although the appearance of the MRI is similar and in which new lesions do not occur. Because of the unusual appearance of these MRI lesions, brain biopsy is often performed but recognising their relevance should obviate that need.
\end{abstract}

(F Neurol Neurosurg Psychiatry 1995;58:467-470)

Division of Neurology, Department of Neurosciences, Riyadh Military Hospital, PO Box 7897, Riyadh 11159 Saudi Arabia $S$ Khan

B A Yaqub

S M Al Deeb

Department of

Neurology, Havard

Medical School, Beth

Israel Hospital, 330

Brookline Avenue,

Boston, MA 02215,

USA

C M Poser

Division of Neurology, Department of Medicine, King Faisal Specialist Hospital and Research Centre, P O Box 3354, Riyadh 11211, Saudi Arabia S Bohlega

Correspondence to:

Dr Sonia Khan, Division of Neurology, Department of Neurology, Department of Clinical Neurosciences, $P$ Saudi Arabia.

Received 28 June 1994 and in revised form 3 October 1994 Accepted 19 October 1994 sclerosis on the basis of the history, the CSF examination, and evoked potential studies but the MRI of the brain favoured the diagnosis of multiphasic disseminated encephalomyelitis (MDEM).

\section{Case reports}

CASE 1

A 32 year old black Sudanese woman was admitted to the hospital because of sudden dysarthria. On the next day, she had a right hemiparesis without impairment of consciousness or seizures. Six years before, while living in the Sudan, she had suddenly had left sided hemiparesis and homonymous hemianopia. The hemiplegia recovered completely in one week, whereas the hemianopia persisted. She was not diabetic or hypertensive, and there was no history of cardiac disease or hyperlipidaemia. Systemic examination was normal. The neurological examination showed normal intellectual function, but she had slurred speech without aphasia. Other findings included left homonymous hemianopia, right upper motor neuron facial nerve palsy, and slight deviation of the tongue to the right without wasting or fasciculations. She had upper motor neuron weakness of the right upper and lower limbs, with hyperreflexia and Babinski sign. There was no limb ataxia or disequilibrium and all modalities of sensations were intact. She had a right hemiplegic gait.

Complete blood count, sedimentation rate, blood urea nitrogen, and serum electrolytes, liver function tests, and autoimmune screen for connective tissue disease, including lupus anticoagulant and phospholipid antibodies, were all normal. Sickle cell screen was negative. The serum titres for brucella, syphilis, and hepatitis $B$ were negative as was the tuberculin skin test. A chest radiograph, ECG, and transesophageal echocardiogram were normal. Doppler examination of the neck vessels and four vessel cerebral arteriography were normal. The CSF showed 2 white blood cells $/ \mathrm{mm}^{3}$, total protein $0.26 \mathrm{~g} / 1$, glucose 3 $\mathrm{mmol} / \mathrm{l}$, and an IgG index of $0 \cdot 6$. No oligoclonal bands were seen. Tests for measles, rubella, mumps, Epstein-Barr, and herpes simplex antibodies and for the presence of fungal antigens were negative. An EEG showed focal slowing in the left frontoparietal and right occipital regions. Pattern shift visual, somatosensory, and brainstem auditory 
evoked potentials were normal. Computed tomography and MRI of the brain showed bilateral multiple, scattered enhancing nodular lesions in the white matter (fig 1a-c).

The diagnoses of multiple tuberculomas or

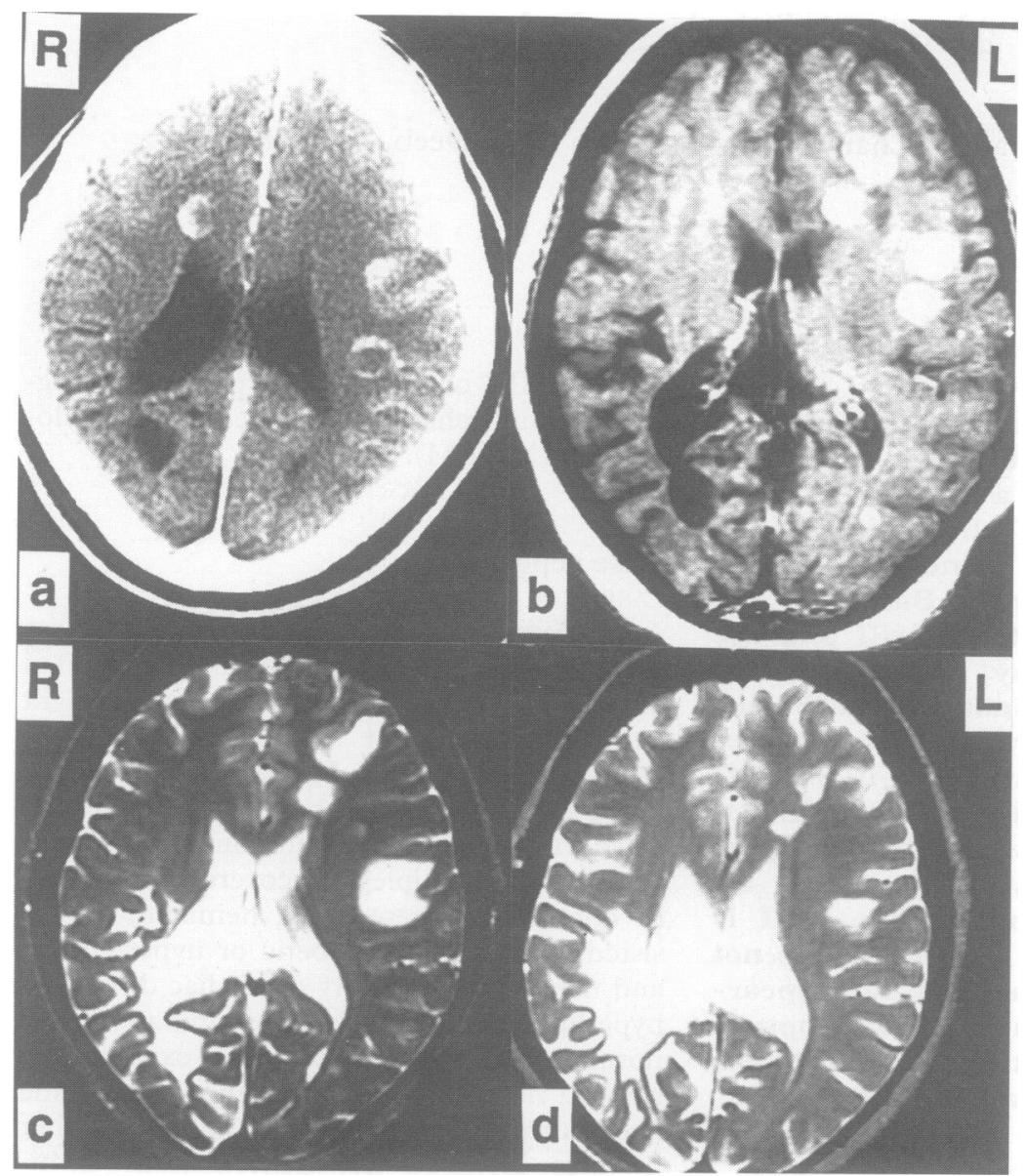

Figure 1 Case 1 (a) Contrast enhanced brain CT: multiple enhancing nodules in both cerebral hemispheres. There is dilatation of the atrium of the right lateral ventricle associated with a hypodense area in the parietotemporal region on the right characteristic of an old lesion. (b) MRI T1 weighted image after gadolinium injection showing five enhancing nodules in the left subcortical white matter. There is a large area of low intensity in the right parieto-occipital region indicating the site of an old, inactive lesion, which probably occurred six years previously when the patient developed left hemianopia and hemiparesis. (c) T2 weighted MRI: multifocal high signal intensity lesions in the white matter of both hemispheres. One of the lesions encroaches on the cortex on the left side. There is extensive white matter high signal intensity in the right parieto-occipital region. Note the sparing of the corpus callosum and the absence of periventricular lesions. (d) $T 2$ weighted MRI eight weeks later: there has been a considerable reduction of the size of the left sided lesions while the one on the right remains unchanged. metastasis were initially considered, but brain biopsy showed inflammatory primary demyelination (fig 2), so a diagnosis of MDEM was made. She was given methylprednisolone $(1 \mathrm{~g}$ intravenously daily for five days), followed by a course of tapering oral steroids. After one week, the hemiparesis started to improve and the dysarthria disappeared after 10 days. Four weeks later, the patient had fully recovered. The hemianopia from her previous episode had remained unchanged. The steroids were discontinued at the end of six weeks. Repeat brain MRI eight weeks after the onset of her illness showed considerable improvement in the white matter lesions (fig 1d), which no longer enhanced after injection of gadolinium.

CASE 2

In June 1990, a previously healthy 29 year old Saudi man had acute right leg weakness, which progressed over a week to involve his right arm and face as well. $\mathrm{He}$ also noted numbness of his right leg. He complained of frontal headache, difficulty in concentrating, and mild weight loss. He had no seizures, fever, diplopia, or urinary difficulties. The neurological examination on admission showed mild dysnomia and right spastic hemiparesis affecting the leg more than the arm and face. There was subjective diminution of pain, temperature, and vibratory sensations. A brain MRI showed two large lesions in the left subcortical white matter and a smaller one posteriorly on the left (fig 3a). There was little adjacent oedema and no mass effect. These lesions enhanced poorly after gadolinium injection. A left internal carotid arteriogram, an EEG, and visual and somatosensory evoked potentials were negative. Blood count, sedimentation rate, electrolytes, autoimmune screen for vasculitis, serological tests for borrelia, brucella, syphilis, cryptococcus, toxoplasma, and HIV were all negative, as were the tuberculin skin test, chest radiograph, ECG, and abdominal ultrasound. A CSF examination showed 6 white blood cells $/ \mathrm{mm},{ }^{3}$ a total protein of 0.37 $\mathrm{g} / \mathrm{l}$, a raised $\mathrm{IgG}$ index of 1.64 (normal $<0.7$ ), IgG synthesis of $19.1 \mathrm{mg} / 24$ hours (normal $0-3 \cdot 5$ ), and three oligoclonal bands.
Figure 2 Case 1: brain biopsy (a) Light microscopy. Perivascular oedema, inflammation, and demyelination

(haematoxylin-eosin). (b) Electronmicrograph showing complete disorganisation of the myelin sheath (white curved arrow on top) and bare axons (white curved arrow and straight black arrow at the bottom). Many macrophages contain myelin bodies within their cytoplasm (two straight white arrows at the bottom right). No viral particles were seen.
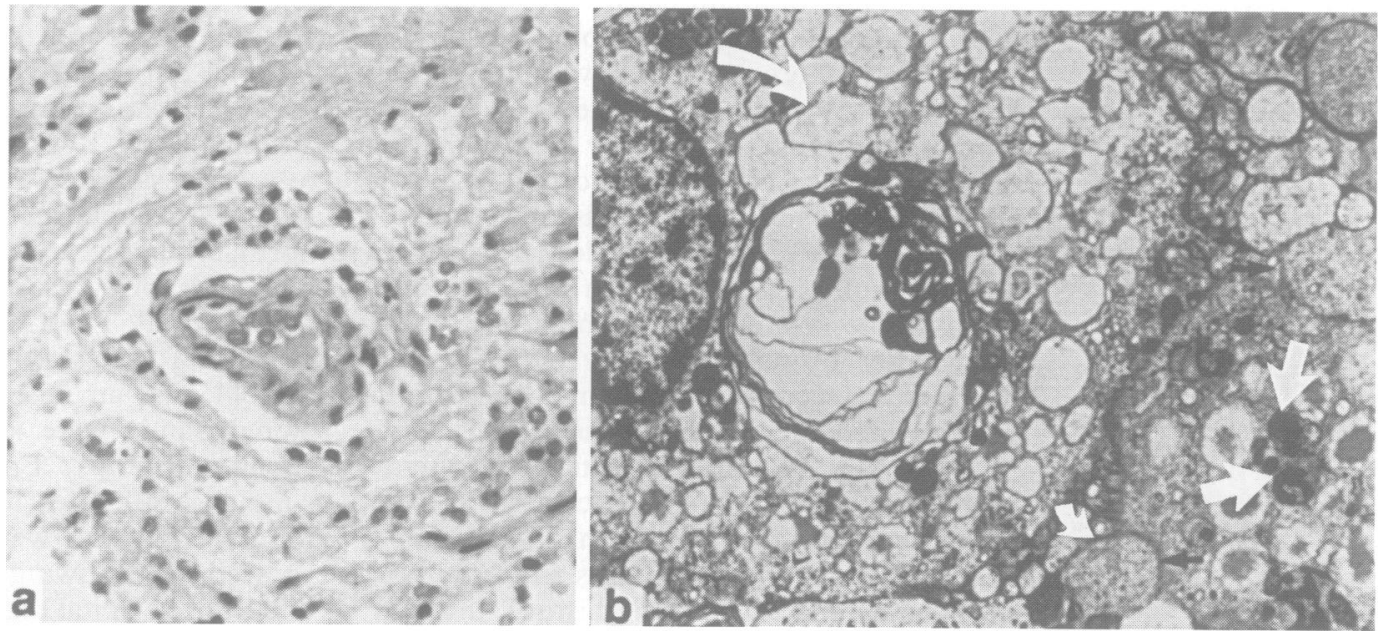


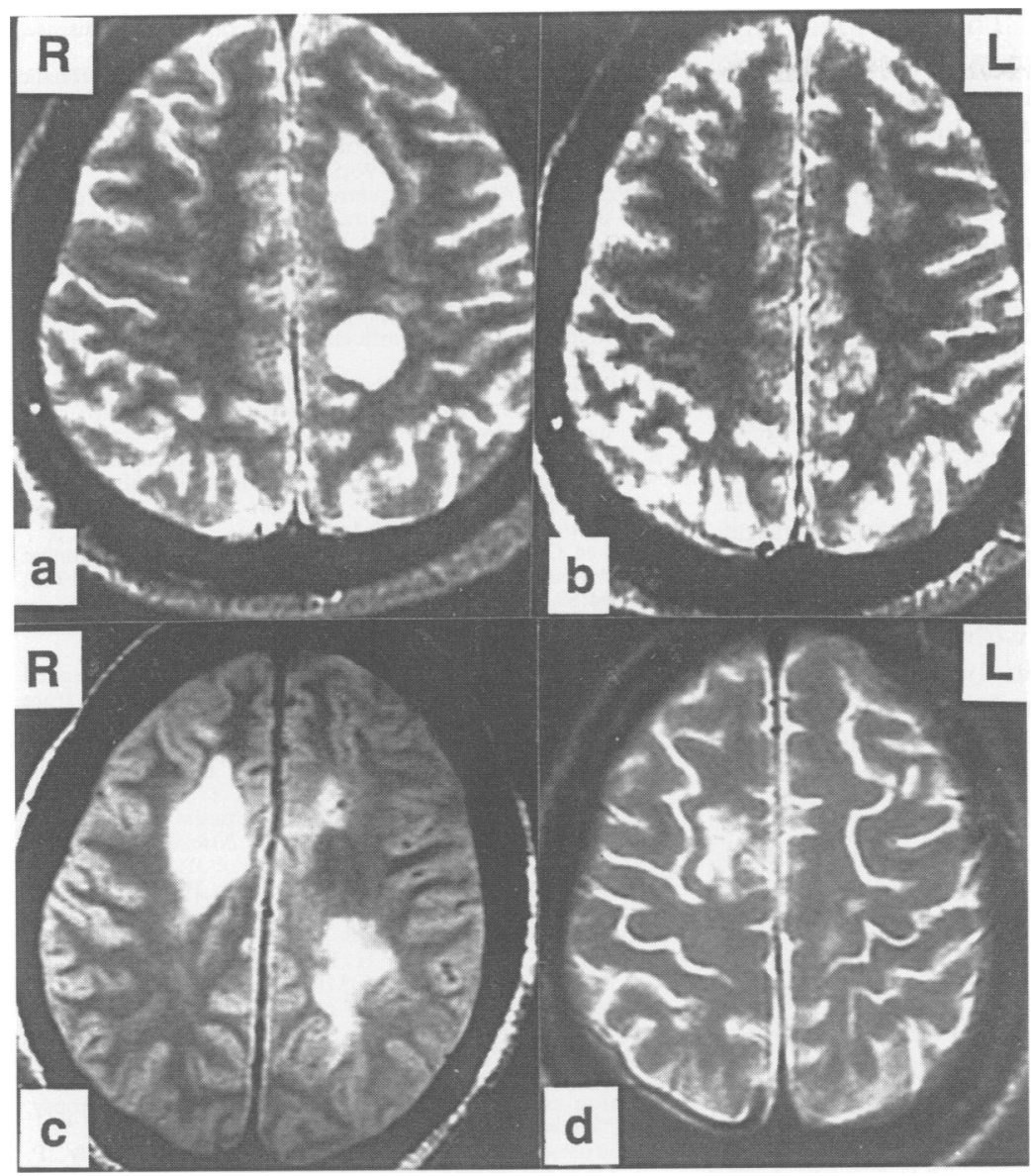

Figure 3 Case 2 (a) T2 weighted MRI: Two very large left hemispheric lesions without mass effect. (b) T2 weighted MRI four months later. Pronounced regression of both lesions. (c) T2 weighted MRI three years later at time of second episode. Large, new lesion in the right frontal area. Note that the previous, left posterior lesion has become much larger and seems to have been reactivated. (d) T2 weighted MRI two months later. Pronounced regression of all lesions. enhanced faintly after gadolinium injection. The patient's weakness improved after treatment with intravenous methylprednisolone. A follow up MRI obtained three months later showed considerable regression of all the lesions (fig 3d).

\section{Discussion}

Classically, acute disseminated encephalomyelitis (ADEM) is a monophasic neurological syndrome that may occur after vaccination against various viruses and after many viral infections. ${ }^{4-7}$ Repeated episodes of postinfectious or postvaccinal neurological syndromes are rare. ${ }^{89}$ Poser $^{10}$ has proposed that disseminated encephalomyelitis (DEM) with multiple episodes be classified into two types. In the first one, an initial episode of ADEM, from which there is complete or partial recovery, is followed by recurrences that are characteristically stereotyped-that is, the symptoms are always the same, although the complete original syndrome is not necessarily present. This is called recurrent DEM (RDEM). RDEM must not be confused with the pseudorecurrences seen in patients with steroid dependent ADEM when treatment is stopped. In the other type, which is represented in these two patients, there are two or more separate acute episodes that differ in clinical presentation, and that is termed multiphasic DEM (MDEM). Both cases of Poser et $a l^{5}$ are examples of MDEM; MDEM is extremely difficult to differentiate from multiple sclerosis on purely clinical grounds, but it is important to do so since the prognosis of MDEM is much better.

In patients with DEM, the CSF most commonly shows a moderate pleocytosis and increase in total protein, although both may be very high. IgG is often increased but oligoclonal bands are rare. Oligoclonal bands never disappear in multiple sclerosis but may do so in DEM, and this is important in differentiating multiple sclerosis from both MDEM and RDEM. ${ }^{11}$ The MRI in DEM is often abnormal and has special characteristics. ${ }^{911-14}$ Although in some cases, the abnormalities cannot be distinguished from those of multiple sclerosis. ${ }^{15}$ Unlike those in multiple sclerosis, the lesions are extensive, often follow the outline of the cortical ribbon, or may consist, as in these two cases, of very large, globular areas of increased signal intensity that do not produce a mass effect. They are often mistaken for neoplasms or abscesses. The cerebellum and cortex are often involved, as are occasionally the thalamus or the basal ganglia. The corpus callosum is usually not affected and the periventricular distribution of the lesions is not as constant. ${ }^{911}$ An important differential point between MDEM and RDEM is that new MRI lesions invariably occur in the first but not in the second. In MDEM, the lesions tend to be of varying age, active lesions can be shown by contrast enhancement on $\mathrm{T} 1$ weighted image, whereas old inactive lesions appear as high signal intensities on T2 weighted image 
as shown in our patients. The large lobar high intensity lesions, which may be seen in DEM, are never seen in multiple sclerosis.

The pathology in DEM is similar to that of multiple sclerosis in showing perivascular oedema, inflammation, and demyelination. The degree of inflammatory reaction will obviously depend on the time elapsed between the onset of the illness and the time of the biopsy. Because the histological differentiation between all types of DEM and multiple sclerosis is based on the topography of the demyelinating process, which is essentially histologically identical in both conditions, it cannot be dependably made on tissue obtained by means of stereotactic needle brain biopsy. In most instances, the characteristic MRI coupled with the history of recurrences, or of a multiphasic illness, mitigates the need for brain biopsy. Sometimes misunderstanding the diagnostic importance of the characteristic MRI appearance of DEM coupled with the results of needle brain biopsy may lead to the erroneous diagnosis of multiple sclerosis. ${ }^{1617}$

As well as the distinctive MRI image, another consideration, albeit a minor one, made us veer away from the diagnosis of multiple sclerosis in case 1: our patient was a black African, in whom the disease is extremely rare, living in Saudi Arabia, an area of low multiple sclerosis incidence. ${ }^{18}$

We thank the members of the audiovisual departments of the hospitals for preparing the photographs, and Ms Divina Nojadera for her excellent secretarial work.
1 Adams $\mathrm{H}$, Biller J. Ischemic cerebrovascular disease. Neurology in Clinical Practice 1991;61:908-37.

2 Biller J, Hingtgen W, Adams H, et al. Cervicocephalic arterial dissections: a ten-year experience. Arch Neurol 1986;43:1234-8

3 Cowan J, Ormerod I, Rudge P. Hemiparetic multiple sclerosis. Neurology 1990;50:675-80.

4 Francis G, Antel J, Duquette P. Inflammatory demyelinating diseases of the central nervous system. Neurology in Clinical Practice 1991;61:1149-65.

5 Poser C, Roman G, Emery E. Recurrent disseminated vasculomyelinopathy. Arch Neurol 1978;35:166-70.

6 Yahr M, Lobo-Antunes J. Relapsing encephalomyelitis following the use of influenza vaccine. Arch Neurol 1972; 27:182-3.

7 Al Deeb S, Yaqub B, Sherif H, Phadke J. Neurobrucellosis: clinical characteristics, diagnosis and outcome. Neurology 1989;39:498-501.

8 Shoji H, Kusuhara T, Honda Y, et al. Relapsing acute disseminated encephalomyelitis associated with chronic seminated encephalomyelitis associated with chronic Epstein-Barr virus infection.
Neuroradiology 1992;34:340-2.

9 Poser C. Magnetic resonance imaging in asymptomatic disseminated vasculomyelinopathy. $\mathcal{F}$ Neurol Sci 1989 94:69-77.

10 Poser C. Multiple sclerosis: clinical aspects and differential diagnosis. Clin Neurosci 1995 (in press)

11 Kesselring J, Miller D, Robb S, et al. Acute disseminated encephalitis. MRI findings and the distinction from multiple sclerosis. Brain 1990;113:291-302.

12 Dunn V, Bale J, Zimmerman R, et al. MRI in children with post infectious disseminated encephalomyelitis. Magn Res Imag 1986;4:25-32.

13 Epperson L, Whitaker J, Kapila A. Cranial MRI in acute disseminated encephalomyelitis. Neurology 1988;38: d32-3.

14 Boulloche J, Parain D, Mallet E, Tron P. Postinfectious encephalitis with multifocal white matter lesions. Neuropaediat $1989 ; 20: 173-5$.

15 Fazekas F, Offenbacher H, Fuchs S, et al. Criteria for an increased specificity of MRI interpretation in elderly subjects with suspected multiple sclerosis. Neurology 1988;38:1822-5.

16 Nesbit G, Forbes G, Scheithauer B, et al. Multiple sclerosis: histopathologic and MR and/or CT correlation in 37 cases at biopsy and three cases at autopsy. Neuroradiology 1991;180:467-74.

17 Rodriguez M, Scheithauer B, Forbes G, Kelly P. Oligodendrocyte injury is an early event in multiple sclerosis. Mayo Clin Proc 1993;68:627-36.

18 Yaqub B, Daif A. Multiple sclerosis in Saudi Arabia. Neurology 1988;38:621-3. 